\title{
Use of Benchmarking Data for Effective Management of Software Development \& Enhancement Projects - Case Studies and the ISO/IEC 29155 Norm
}

\author{
Beata Czarnacka-Chrobot
}

\begin{abstract}
The Software Development \& Enhancement Projects (SD\&EP) management is an area of software engineering that is not proving to be particularly highly effective. One of the major effects of this status quo is a waste of considerable sums of money having been invested in projects of this type. While one of the major causes is inability to plan duration and costs of the realization of such projects. On the other hand, this results, among others, from the fact that collecting and use of reliable benchmarking data that would provide possibility to determine dependence between the project's product size and work effort and its duration in specified realization conditions, is still rare. The goal of this paper is to demonstrate several examples of case studies employing such data, for which these data contributed to the increase in the effectiveness of management of the discussed projects, executed in public administration institutions. The paper also analyzes the source with general benchmarking data, being employed in these case studies, as well as it points to the need to normalize data sources of this type according to the ISO/IEC 29155 norm, just being developed. )
\end{abstract}

Keywords-software development and enhancement projects, estimation, benchmarking data, ISO/IEC 29155, case studies

\section{Introduction}

Spendings on Software Development \& Enhancement Projects (SD\&EP) may considerably exceed the expense of building offices occupied by companies commissioning them, and in extreme cases, even 50-storey skyscraper, roofed football stadium, or cruising ship with a displacement of 70.000 tons [1]. Meanwhile, as indicated by the results of the Standish Group studies [2], success rate for such projects has never gone beyond $31 \%$. Right now, out of 100 completed software projects, 71 end up as unsuccessful projects - among them 19 are failed projects while on the remaining 52 projects one has to spend on average about $150 \%$ of the estimated costs, and their completion may be expected after about $170 \%$ of the planned time on average. The end product on average delivers only not more than $70 \%$ of the required functions and features. The Panorama Consulting Group, when investigating in their 2015 study [3] the effectiveness of ERP (Enterprise Resource Planning) systems projects being accomplished worldwide revealed that "over the past five years, the average cost of ERP implementation has been approx. \$6,1 million with an average duration of 15,7 months." Approximately 58\% of ERP projects exceeded their planned costs, $65 \%$ of them overrun their estimated time. What's more, 53\% of investigated organizations declared, that they achieved less than $50 \%$ of the expected benefits from the ERP implementation. Last year more than year ago such projects experienced duration overrun $(75 \%$ vs. $72 \%)$ and cost overrun $(55 \%$ vs. $54 \%)$. While nearly half of organizations (45-percent) reported that their ERP projects were on or under budget, the number of reported ERP failures increased by five-percent since that time.

Unsuccessful software projects, especially with their high costs being considered, lead to a substantial financial losses, on a worldwide scale estimated to be hundreds of billions of dollars yearly, sometimes making even more than half the funds being invested in such projects. The Standish Group estimates that these losses - excluding losses caused by business opportunities lost by clients, providers losing credibility, or legal repercussions - range, depending on the year considered, from approx. $20 \%$ to even $55 \%$ of the costs assigned for the execution of the analyzed project types. Analyses of the Economist Intelligence Unit indicate that there is a strong correlation between delays in delivery of software products and services and decrease in profitability of a company therefore failures of projects, resulting in delays in making new product and services available and in decreasing the expected income, represent threat also to the company's business activity [4].

Cited research results are proof that the execution of SD\&EP is not sufficiently effective, and this is because the management of the process too rarely meets one of the criteria for a rational investment decision, namely the criterion of effectiveness, meaning that such decision should contribute to achieving the assumed result, in the case of SD\&EP usually being considered as delivering product meeting client's requirements with regard to functions and features without budget and time overruns (see e.g., [5]). Effective management of SD\&EP makes serious difficulties as objective and reliable cost and duration estimation still appears to be a great challenge to the software engineering. Thus rational pricing of software as a product of such projects, being of key significance to clients, encounters serious problems in practice. The above situation manifests itself in the difference in costs spent by various organizations on similar applications that may be even fifteen fold [6].

In the author's opinion the main reason of that problem is wrong project work effort estimation - on the basis of resources, while such activity should ground on the required software size, which in turn should result directly from the user's requirements. 
Work effort, costs, duration of projects, and therefore the productivity (understood as a relationship between the size of the software to work effort) are possible to estimate using appropriate benchmarking data. This data should allow to predict these SD\&EP attributes based on the expected size of the product (software) and on the specific assumptions.

The paper consists of 5 sections. The main goal of this paper is to demonstrate several examples of case studies employing benchmarking data, for which these data contributed to the increase in the effectiveness of management of the discussed projects, executed in public administration institutions, what can be read in Chapter 3 . But after Introduction, in Chapter 2 it first analyzes the source with general benchmarking data, being employed in these case studies. In Chapter 4 it points also to the need to normalize data sources of this type according to the ISO/IEC 29155 norm, just being developed. Concluding remarks are included in Chapter 5.

\section{Sources of Software Benchmarking Data}

Sources of software benchmarks are "hunted" by Capers Jones [7]. In 2014 he distinguished 24 sources of software benchmarking data from more than 90 thousand software projects yet majority of them are not widely available. These sources gather differentiated quantitative data that is collected with the aim of improving the software and projects measurement, calibration of estimation tools, but also to improve the quality of software. These data are collected by firms, "non-profit" groups, universities and similar organizations with a view to their use by software managers and engineers. The largest, commonly recognized and widely available repository containing general benchmarking data for improvement of SD\&EP measurement, whose products are measured using the Functional Size Measurement (FSM) methods [8], is managed by the International Software Benchmarking Standards Group (ISBSG) [9].

The ISBSG is a non-profit organization founded in the second half of the 1990s. with the purpose to enhance the processes of IT resources management both in business entities as well as in public administration institutions. This goal is being fulfilled by developing, maintaining and using several repositories with benchmarking data. One of them, the biggest one (current version of this repository contains data concerning more than 7500 projects from about 30 countries) includes data for completed software development and enhancement projects [10]. It is normalized according to the ISO/IEC 15939 standard [11], verified and representative of the current technology.

Data gathered in the discussed repository have been classified by the ISBSG with regard to the following criteria [12]: (1) country of effort; (2) context of project, including type of organization and area of business; (3) type of project, including type of actions (modification/enhancement, development from scratch), purpose of project (internal or external needs) and size of development team; (4) type of product, including type of application and product size; (5) project execution environment, including programming language and hardware platform; (6) development methods and tools being used.

Using data gathered by this organization one should bear in mind that they are representative of rather above-average projects which results from the following (for more details see [13]): (1) criteria of gathering data in ISBSG repository take into account only those organizations that are using FSM methods while such organizations are considered more mature than others because they execute programs concerning implementation of software measures; (2) this is developers themselves that choose projects whose data they provide to the ISBSG repository - those may include projects that are typical of them as well as projects having best parameters; (3) the ISBSG repository does not feature too many data about very large projects. The ISBSG data are subject to rigorous process of verification with regard to quality. Hence they are appreciated in the IT industry while general conclusions coming from their analysis correspond with the conclusions coming from the SD\&EP.

The ISBSG repository is very useful, because relatively few development organizations possess appropriate resources of own benchmarking data as the condition to have them is not only effective implementation of measurement programs, what per se is not a frequently found phenomenon, but having collected such data for relatively large number of similar projects having been executed in the past and, additionally, referring them to the right unit of software system size.

ISBSG is an organization whose activities and experience had a significant impact to the ISO/IEC 29155 norm, although its repositories using in the case studies mentioned below - inevitably - has not yet formed in accordance with this standard.

\section{Using Benchmarking Data - Case Studies}

\section{A. What Is the Cost of One IFPUG Method Function Point?}

This case study applies to the tender competition concerning enhancement of dedicated software system of specific public administration institution in Poland where one of the two potential developers offered possibility to modify such system at the cost of 1 cent per 1 Function Point (FP) of the International Function Point Users Group (IFPUG) method [14], whereas another one attempted to prove that enhancement at such unit cost was not possible to carry out. The goal of this case study was to analyze likely per-unit costs of the software enhancement with regard to 1 IFPUG FP.

Per-unit costs of the SD\&EP are difficult to estimate if a provider of the dedicated system does not have at their disposal their own resources of appropriate benchmarking data, on the basis of which they would be able to determine their own (organizational) per-unit costs with regard to 1 IFPUG FP. This results from the fact that such data depend on a number of specific factors - on a general level including first of all work costs that vary from country to country as well as type of project, type of software system, field of system application and technological environment of 
project execution as well as many other factors having an effect on a large differentiation of development teams productivity.

The ISBSG produces cyclical analytical reports based on the data concerning SD\&EP. What appears of significance from the perspective of the subject matter being discussed in this case study is the ISBSG report titled "Software Project Costs" [15], which analyzes the size of per-unit costs with regard to 1 IPFUG FP. Data analyzed therein indicate that:

- $\quad$ For definite majority of cases, per-unit costs measured with regard to the 1 IFPUG FP range from USD 300 to USD 1000, with an average of about USD 750 per 1 FP. Taking into account all analyzed projects, the spread is from USD 17 to USD 2727 per 1 FP while the cost median is USD 716 per $1 \mathrm{FP}$. These costs are measured by taking into account development team and support personnel (e.g., data base administrators) - they are approx. $15 \%$ higher than costs estimated for development teams only.

- For definite majority of projects, per-unit costs measured with regard to the work time unit ( 1 hour) range from USD 60 to USD 105, with an average of about USD 80 per hour. Taking into account all analyzed projects, the spread is from USD 7 to USD 570 per hour while the cost median is USD 69 per hour and the mean is USD 84 per hour. As in the previous case, these are costs measured with development team and support personnel being taken into account.

On the basis of the above, the ISBSG recommends employing the following rules of thumb for the discussed projects:

1) cost per 1 IFPUG FP ranges from USD 300 to USD 1000, with an average of about USD 750 per $1 \mathrm{FP}$,

2) cost per 1 hour ranges from USD 60 USD to USD 105, with an average of about USD 80 per 1 hour.

What is more, the ISBSG data indicate that PDR (Project Delivery Rate - the inverse of productivity) median, that is middle value of the number of person-hours necessary to deliver 1 IFPUG FP, ranges from about 8 to 11 person-hours per $1 \mathrm{FP}$ - mainly depending on the project type, software system (product) type, application area and technology (in this case median is a value more reliable than arithmetic mean as the impact of several atypical, outlier projects is thus avoided). Besides, productivity is significantly lower (that is PDR is higher) in case of projects consisting in enhancement of software systems rather than in case of projects consisting in developing such systems from scratch [16]. Taking into account those values together with the cost per hour gives us the spread of costs from USD 480 per $1 \mathrm{FP}$ to USD 1155 per $1 \mathrm{FP}$, that is on average from USD 640 to USD 880 per $1 \mathrm{FP}$, which roughly confirms the conclusions coming from the above analysis of the unit cost per 1 IFPUG FP.

Moreover, if project is executed by an outside provider, one should differentiate internal per-unit costs (provider's per-unit work costs) from external ones (per-unit costs offered by provider to a client, including profit as well). According to the ISBSG, the latter usually exceed internal per-unit costs by 2,5 to 3 times, and in big corporations even by 6 times [17, p. 128].
Thus it should be stated that general data from ISBSG repository (and even those coming from other sources having been recognized in the IT industry), as well as common sense rules of rational economic approach unequivocally indicate that it is not possible to develop, and in particular to enhance software system dedicated to the client's needs at the cost per unit amounting to 1 cent per 1 IFPUG FP, at the same time assuming the lack of subsidization for those works with maintenance costs or other project-related costs, which naturally should not have happened.

This analysis resulted in client rejecting the provider offering such costs in the tender competition being considered (for more details about this case study see [18]).

\section{B. Analysis of the Workflow System Enhancement Project Speed}

This case study applies to the tender competition concerning the enhancement of Workflow System (WS) of one of the public institutions in Poland in which one of the two potential developers offered a possibility to enhance such system at the project speed of 0,8 Function Point of the Common Software Measurement International Consortium (COSMIC) method [19] per one hour, whereas the other one attempted to prove that such project speed is overestimated. The criterion of project speed, being one of the three criteria considered, determined client's decision on selecting developer offering that particular value. The goal of this case study is to demonstrate if it is possible to carry out the WS enhancement at the above mentioned project speed (and, consequently, within the project duration resulting from that attribute). The analysis served as a main basis for settling legal dispute between a company offering values of attributes that are being analyzed and the competing company.

Project speed is a quotient of project's software product size to the duration of product delivery, that is in this case it is understood as the number of the COSMIC Function Points (CFP) delivered within a certain time, e.g., 1 hour $(\mathrm{CFP} / \mathrm{h})$. Project speed is difficult to determine since it depends on a number of factors. Primarily it depends on the type of project, type and size of project's product (software) as well as technological environment of project execution, mainly on the generation of programming languages being used.

As indicated by the documentation provided by a client commissioning analysis, the subject of considerations in this case study is project speed for which the following factors affecting its value should be taken into account: (1) size of changes of the Workflow System up to 4000 CFP; (2) the Workflow System has a character of business application (i.e., being data-driven); (3) the Workflow System is dedicated to the client's needs; (4) undertakings within the area of interest of this analysis consists in software extension (enhancement project); (5) for development activities, the third Generation Languages (3GL) will be used.

Based on the above assumptions, certain company offered project speed of 0,8 CFP per 1 hour, with the size of project development team being as required by the client, that is 10 persons. Thus the company believes it is possible 
to deliver not less than 0,8 CFP within the time of 1 hour by the team consisting of 10 persons.

Based on data concerning dedicated SD\&EP the ISBSG produces cyclic analytical reports. From the perspective of the issues discussed in this paper the most important is the ISBSG report made in collaboration with the COSMIC, i.e., "The Performance of Business Application, Real-Time and Component Software Projects. An analysis of COSMIC measured projects in the ISBSG database" [20], which analyses, among others, speed of delivering COSMIC function points for various types of software systems, including their sizes, depending on the type of project and generation of programming language. What is of our interest here is project speed of dedicated business applications enhancement with the use of $3 \mathrm{GL}$ programming language.

In case of business application enhancement projects, the following regularities prove characteristic - they were identified on the basis of all 76 projects, i.e., without dividing them by the criterion of the generation of programming language being used; there were 67 projects analyzed for applications in the 3GL and 9 projects for 4GL applications ([20, pp. 20-21]): project speed (PS) for delivery of COSMIC function points during a month is calculated according to the below formula:

$$
\mathrm{PS}=2,34 \mathrm{x}(\text { size in CFP })^{0,40}
$$

Having assumed the size of 4000 CFP we obtain:

$$
\mathrm{PS}=64,57 \mathrm{CFP} / \mathrm{month},
$$

i.e., taking into account conversion rate set by the client where the number of working days during one month is 21 days whereas each working day is 8 hours we obtain the following:

$$
\mathrm{PS}=3,07 \mathrm{CFP} / \text { day and } \mathrm{PS}=0,38 \mathrm{CFP} / \text { hour, }
$$

which implies PS being twice lower than the offered one.

Taking into account assumptions adopted in the analysis and regularities resulting from the ISBSG benchmarking data it should be stated that project speed in case of dedicated business application enhancement project of the size of 4000 CFP should oscillate around 0,38 CFP per hour.

In the analyzed report [20] it is also stated that, generally speaking, for application sizes above 100 CFP there are no significant differences with regard to the project speed between application enhancement projects and application development projects (below that size there is a difference in favour of project speed for application enhancement projects). Therefore, suggestion of the authors of the report made with regard to the analysis of the per-unit work effort, indicating that large business application enhancement projects (i.e., those having application size above 1000 CFP) should be treated as projects of their development may not apply to the project speed; also there are no statistically adequate data that would allow to determine the effect of particular impact factors on project speed.

However, to keep up the objectivity of our analysis it is worth to bring up data concerning projects consisting in development of dedicated business applications. In this case projects employing 3GL and 4GL programming languages were analyzed separately. Data presented in the discussed report indicate that for business application development projects using $3 \mathrm{GL}$ programming languages (77 among the analyzed projects) particular regularities read as follows ([20, pp. 13-16]): project speed (PS) for delivery of COSMIC function points per month:

$$
\mathrm{PS}=0,31 \mathrm{x}(\text { size in CFP })^{0,67} \text {. }
$$

Having assumed the size of $4000 \mathrm{CFP}$ it then reads as follows:

$$
\mathrm{PS}=80,30 \mathrm{CFP} / \mathrm{month},
$$

i.e., taking into account conversion rate set by the client where the number of working days during one month is 21 days whereas each working day is 8 hours we obtain the following:

$$
\mathrm{PS}=3,82 \mathrm{CFP} / \text { day and PS }=0,48 \mathrm{CFP} / \text { hour, }
$$

which means PS being nearly $70 \%$ lower that the offered one.

Taking into account assumptions adopted in the analysis and regularities resulting from the ISBSG benchmarking data it should be stated that project speed in case of dedicated business application development project of the size of 4000 CFP should oscillate around 0,48 CFP per hour.

Summing up it should be stated that from the rational point of view, taking into account assumptions adopted in this analysis as well as the above presented regularities resulting from the objective benchmarking data, the offered Workflow System enhancement project speed on the level of 0,8 CFP per 1 hour should be regarded as overestimated (for more details about this case study see [21]).

\section{The ISO/IEC 29155 Standard}

„Benchmarking is an activity of comparing "object of interest" to each other or against a benchmark to evaluate characteristic(s). In the context of the ISO/IEC 29155-series, the "object of interest" is the performance of information technology (IT) project, and the characteristic is a particular aspect of an IT project such as productivity. The benchmarking is considered to be one of the fastest-growing techniques in the area of IT project management." [22]. The most important reasons for its implementation include the need to: (1) compare project productivity between similar industries; (2) compare productivity between different project types and technologies; (3) find the most effective targets for IT development/enhancement process improvement; (4) compare productivity between different suppliers; (5) improve project management maturity, and (6) improve project estimation capability. In order to standardize the actions to implement these requirements the ISO/IEC 29155 standard was established.

The ISO/IEC 29155 norm is composed of four parts:

1) Part 1: „Concepts and definitions” [23] - it contains, in addition to the basic concepts and definitions, above all a framework for IT project performance benchmarking, which includes activities and components necessary to identify, define, select, use and improve of benchmarking for IT project performance (for data selection and comparison). It is dedicated to the stakeholders of IT project performance benchmarking.

2) Part 2: „Requirements for benchmarking” [24] - it contains the general requirements for the processes of the IT project performance benchmarking, i.e., describes: (a) the 
steps required to properly identify, define, select, apply, and improve benchmarking for IT project performance (e.g., conduct benchmarking, maintain repository, submit data); (b) the tasks necessary for the proper implementation of these activities and to equip them with the components. It is dedicated to be used mainly by organizations that are preparing for the introduction of techniques for benchmarking to evaluate the performance of IT projects. It is useful for benchmarking user, the benchmark provider, benchmarking service provider, and IT project team.

3) Part 3: „Guidance for reporting” [25] - it contains general requirements and guide for: (a) reporting processes and (b) the contents of typical reports within benchmarking activities of IT project performance benchmarking framework. This part focuses on three main activities: conduct benchmarking, maintain repository, and issue benchmarks. These activities are crucial because of its result, which is a fundamental value for benchmarking users. This part focuses on two major reports: (a) the report describing the results of an instance of benchmarking, (b) the explanatory report, containing the necessary information about the released benchmarking repository or benchmarks. It is therefore intended primarily for: (a) benchmarking user, (b) systems/software acquirer to define, order, obtain and evaluate a benchmarking report, (c) benchmark provider to provide complementary information about the released benchmarking repository or issued benchmarks, and (d) benchmarking service provider to produce a high-quality benchmarking report.

4) Part 4: "Guidance for data collection and maintenance" [26] - it contains the general requirements and guidance for: (a) data element definitions, (b) the data collection and maintenance processes within the benchmarking framework, and (c) delivering the benchmarking repository within benchmarking activities by maintaining benchmarking repository product and issued benchmarks. The three main activities covered by this part of the standard are: maintaining repository, submitting IT project data, and IT project measurement. It will be designed to: (a) benchmarking user, including benchmarking analyst to use benchmarking repository product and/or benchmarks for executing an instance of benchmarking, (b) benchmark provider to define data elements, collect and maintain IT project data, and provide benchmarking repository product or issued benchmarks, (c) benchmarking service provider to utilize benchmarking repository product and/or benchmarks for providing benchmarking services, (d) IT service provider to define data elements to be measured and/or to be submitted to repository owner, and (e) IT project team.

The ISO/IEC 29155 standard is strongly associated with other standards, including in particular: project management (e.g., PMBOK and ISO 10006), systems and software measurements (e.g., ISO/IEC 15939), software life cycle processes (e.g., ISO/IEC 12207), systems life cycle processes (e.g., ISO/IEC 15288), functional size measurement (e.g., ISO/IEC 14143 series and related methods), systems and software quality evaluations (e.g., ISO/IEC 25000 family and ISO/IEC 9126 series). However, the starting point for the ISO/IEC 29155 series was the concept outlined by the ISBSG.

\section{v. Concluding Remarks}

The paper presented the possibilities of improving the effectiveness of SD\&EP management in public administration institutions by usage of IT benchmarking data on some examples concerning the real situations.

One of the reasons of low success rate for SD\&EP is inaccurate estimation of their attributes: in project practice there is a tendency for estimation to be overly optimistic and therefore these attributes in fact are often being exceeded. In the author's opinion the main reason for this is estimation on the basis of wrong methods. Empirical research and international standards indicate that for estimation of SD\&EP attributes, methods based on software functional size measurement may be regarded good enough. However, reliable estimation of project effort, cost, duration, productivity, speed (and others) based on the software functional size requires benchmarking data, on the basis of which it is possible to derive necessary dependencies.

General benchmarking data repositories are designed to develop dependencies of indicative character, which are not sufficient for formal valuation of products, require a lot of caution when using them as well as awareness of the fact that they reflect dependencies existing in the group of projects of given specificity, but are useful in the situation of the lack of own organizational benchmarking data. That's why software houses and their clients, e.g., public administration institutions, should develop their own repositories. However, the proper usage of benchmarking data will be possible on the condition that repositories containing them will be extended according to the rules of the ISO/IEC 29155 standard.

\section{References}

[1] T.C. Jones, "Software Project Management in the Twenty-first Century", SPR, Burlington, 1999.

[2] Standish Group, "Chaos Manifesto 2015”, Standish Group, West Yarmouth, Massachusetts, 2015.

[3] PCG, "ERP Report”, Panorama Consulting Group, Denver, 2015.

[4] Economist Intelligence Unit, "Global survey reveals late IT projects linked to lower profits, poor business outcomes", http://www.hp.com/hpinfo/newsroom/press/2007/070605xa.html (14.06.2013).

[5] B. Czarnacka-Chrobot, "Rationalization of Business Software Systems Development and Enhancement Projects Investment Decisions on the basis of Functional Size Measurement", in International Journal of Software Engineering and Knowledge Engineering, vol. 23, no. 06, World Scientific Publishing, USA, 2013, pp. 839-868 (JCR); DOI: 10.1142/S0218194013500228.

[6] State Government of Victoria, "southernSCOPE, Reference Manual," Version 1, Government of Victoria, Melbourne, September 2000

[7] NESMA, http://nesma.org/themes/benchmarking/sources-ofbenchmark-data/ (16.10.2016).

[8] ISO/IEC 14143 Information technology - Software measurement Functional size measurement - Parts 1-6. ISO, Geneva, 2002-2012.

[9] ISBSG, http://www.isbsg.org (10.06.2016)

[10] ISBSG, http://isbsg.org/2016/04/24/release-2016-r1-of-the-isbsgdevelopment-and-enhancement-repository-is-now-available (10.06.2016)

[11] ISO/IEC 15939:2007 Systems and software engineering Measurement process, ISO, Geneva 2002-2007.

[12] ISBSG, "Data demographics, release 11," ISBSG, Hawthorn, VIC, June 2009.

[13] B. Czarnacka-Chrobot, "The role of benchmarking data in the software development and enhancement projects effort planning", in New Trends in Software Methodologies, Tools and Techniques, H. Fujita, V. Marik (Eds.), vol. 199, IOS Press, Amsterdam-BerlinTokyo-Washington 2009, s. 106-127. 
Proc. of the Fourth International Conference on Advances in Computing, Electronics and Communication - ACEC 2016.

Copyright (C) Institute of Research Engineers and Doctors. All rights reserved.

ISBN: 978-1-63248-113-9 doi: 10.15224/ 978-1-63248-113-9-04

[14] ISO/IEC 20926:2009 Software and systems engineering -- Software measurement -- IFPUG functional size measurement method 2009. ISO, Geneva, 2009.

[15] ISBSG, "The ISBSG Special Analysis Report: Software Project Costs", ISBSG, Hawthorn, VIC, June 2005.

[16] Ch. Symons, "The Performance of real-time, business application and component software projects", COSMIC and ISBSG, September 2009.

[17] "Practical Software Project Estimation", P.R. Hill, Ed., ISBSG, McGraw-Hill, 2010.

[18] B. Czarnacka-Chrobot, "What Is the Cost of One IFPUG Method Function Point? Case Study", Proceedings of the 11th International Conference on Software Engineering Research and Practice (SERP'12), The 2012 World Congress in Computer Science, Computer Engineering \& Applied Computing (WORLDCOMP'12), Hamid R. Arabnia, Hassan Reza, Jay Xiong (eds.), CSREA Press, Las Vegas, Nevada, USA, July 2012, pp. 278-283 (http://worldcompproceedings.com/proc/p2012/SER2400.pdf).

[19] ISO/IEC 19761:2011 Software engineering -- COSMIC: a functional size measurement method. ISO, Geneva, 2011.

[20] H. Heeringen and Ch. Symons, "The performance of business application, real-time and component software projects. an analysis of COSMIC-measured projects in the ISBSG database," COSMIC and ISBSG, March 2012.

[21] B. Czarnacka-Chrobot, "Analysis of the Workflow System Ehancement Project Speed and Duration - a Case Study", in Software Engineering", vol. 4, no. 1, Scientific \& Academic Publishing, Rosemead, California, USA, 2014, pp. 10-18; DOI: 10.5923/j.se.20140401.02.

[22] ISO, https://www.iso.org/obp/ui/\#iso:std:iso-iec:29155:-3:ed-1:v1:en (16.10.2016)

[23] ISO/IEC 29155-1:2011, Systems and software engineering Information technology project performance benchmarking framework - Part 1: Concepts and definitions, ISO, Geneva 2011.

[24] ISO/IEC 29155-2:2013, Systems and software engineering Information technology project performance benchmarking framework - Part 2: Requirements for benchmarking, ISO, Geneva 2013.

[25] ISO/IEC 29155-3:2015, Systems and software engineering Information technology project performance benchmarking framework - Part 3: Guidance for reporting, ISO, Geneva, 2015.

[26] ISO/IEC 29155-4:2016, Systems and software engineering Information technology project performance benchmarking framework - Part 4: Guidance for data collection and maintenance, ISO, Geneva, 2016.
About Author (s)

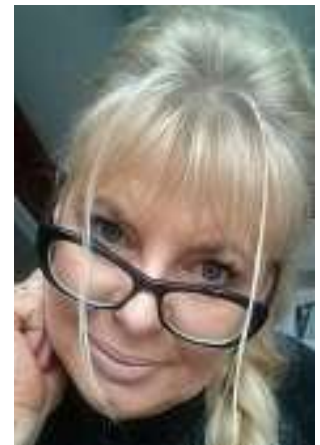

Prof. Beata Czarnacka-Chrobot is chairman of the Scientific Policy Committee at the Polish Ministry of Science and Higher Education, vicedean of the Collegium of Economic Analysis at Warsaw School of Economics (WSE), Poland, head of the Unit of Information Technology at the Institute of Information Systems and Digital Economy in WSE. She is also a member of Polish-United States Innovation Council and one of the Polish representatives of Common Software Measurement International Consortium (COSMIC) International Advisory Council, as well as a vicepresident of the Polish Association for Software Measurement and a member of many others professional organizations. Her research interests relate first of all to the software engineering, especially in the area of software products and processes measurement. She has devoted to this issue the vast majority of the approx. 100 of her publications, including articles published in recognized international journals (with impact factor) and conference proceedings, including some identified as the "Best Paper" of the prestigious IT conferences (the publications list: http://czarnackachrobot.info/?page_id=16). She also works as an expert/advisor/consultant in public administration institutions, consulting firms and IT sector as well as an expert/reviewer for the National Science Centre and the European Commission in FP7, EACEA 2007 and 2013, and Horizon 2020 in the area of ICT. 